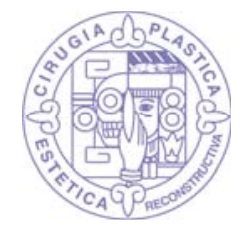

\title{
Sensibilidad glútea posterior a lipoinyección glútea
}

\author{
Gluteal sensitivity after gluteal lipoinjection \\ Dr. André Víctor Baldin,* Dr. Jorge Raúl Carrillo Córdova,* Dr. José Eduardo Telich-Tarriba,* \\ Dr. Gerardo Chávez Pérez,* Dr. Alexander Cárdenas Mejía**
}

Palabras clave: Glúteo, lipoinyección, insensibilidad, lesiones nerviosas.

Key words: Gluteus, lipoinjection, insensitivity, nerve injuries.

\footnotetext{
* Residente de Cirugía Plástica y Reconstructiva. ** Jefe de la Clínica de Parálisis Facial y Nervio Periférico.
}

Hospital General «Dr. Manuel Gea González».

Los autores de este artículo no tienen conflicto de intereses qué declarar.

Recibido:

13 noviembre 2017

Aceptado para publicar:

5 mayo 2018

\section{RESUMEN}

La exhibición corporal, específicamente de la región glútea, ha favorecido la búsqueda cada vez más grande de lipoinyección para embellecer esa zona. A lo largo del tiempo se han descrito diversas técnicas de lipoinyección; sin embargo, la información del postoperatorio es limitada, sobre todo cuando se habla de sensibilidad, siendo ésta un punto fundamental. Realizamos un estudio transversal para evaluar la sensibilidad glútea después de la lipoinyección glútea. Incluimos a 20 pacientes en dos grupos de 10 cada uno; el primero sometidos a liposucción y el segundo sin liposucción. La región glútea se dividió en cuatro cuadrantes y se evaluó la sensibilidad en cada uno de ellos. Los resultados mostraron en el primer grupo a dos pacientes con insensibilidad al roce: una en el cuadrante superomedial izquierdo y la otra insensibilidad al roce en la región superolateral derecha. En el segundo grupo una paciente tuvo insensibilidad al roce en el cuadrante inferomedial derecho. Los demás parámetros evaluados fueron positivos en todas las pacientes. Concluimos que la insensibilidad glútea después de un procedimiento estético de lipoinyección en esa región se debe prever y plantear como una posible complicación, misma que puede traer consecuencias catastróficas en la paciente y en el cirujano.

\section{INTRODUCCIÓN}

T a belleza del cuerpo ha evolucionado en Ltérminos de percepción de la región glútea. En la actualidad, el aumento del volumen de los glúteos representa un área de estudio a considerar, ya que se ha asociado como un sinónimo de femineidad y se le considera como un elemento esencial en la composición de la belleza corporal. La lipoinyección glútea es actualmente el «estándar de oro» para obtener un aumento de esta región anatómica. ${ }^{1,2}$

La cirugía plástica mexicana juega un papel fundamental en la historia de la cirugía glútea. González-Ulloa ${ }^{3}$ es considerado como uno de los grandes precursores de la gluteoplastia de

\section{ABSTRACT}

Body exhibition, specifically of the gluteal region, has favored the increasingly large search for lipoinjection to beautify that area. Over time, several lipoinjection techniques have been described; however, postoperative information is limited, especially regarding sensitivity, this being a fundamental point. We carried out a crosssectional study to evaluate gluteal sensitivity after gluteal lipoinjection. We included 20 patients in two groups of 10 each; the first undergoing liposuction and the second without liposuction. The gluteal region was divided into four quadrants and the sensitivity in each of them was evaluated. The results showed two patients in the first group with insensitivity to rubbing: one in the left upper-medial quadrant and the other insensitivity to rubbing in the right upper-lateral region. In the second group, one patient had insensitivity to rubbing in the right inferior medial quadrant. The other parameters evaluated were positive in all patients. We conclude that gluteal insensitivity after an aesthetic procedure of lipoinjection in this region should be anticipated and posed as a possible complication, which can have catastrophic consequences for the patient and the surgeon. aumento: propuso la utilización de implantes subcutáneos. Más tarde, De la Peña, ${ }^{4}$ describe la utilización del plano subfascial; sin embargo, debido a las complicaciones inherentes de los implantes glúteos como infección, mala posición de los implantes y exposición de los mismos, éstos son indicados para un grupo reducido de pacientes.

El inicio de la liposucción y sus avances han revolucionado el tratamiento del contorno corporal y en la actualidad se propone el uso de la grasa obtenida como un elemento clave para lograr el aumento de la región glútea de manera segura y práctica. ${ }^{5,6}$

Existen diversas técnicas para efectuar la lipoinyección glútea; empero, el manejo de 
la información durante el postoperatorio está limitado. Uno de los aspectos más importantes que se deben estudiar es la sensibilidad: su estudio es un factor fundamental. 7,8

\section{MATERIAL Y MÉTODO}

Realizamos un estudio transversal para investigar la presencia de alteraciones en la sensibilidad en pacientes sometidos a lipoinyección glútea. La muestra incluyó a 20 pacientes sometidos a liposucción, divididos en dos grupos de 10 pacientes cada uno que fueron evaluados a los seis meses del procedimiento. Al primer grupo se sumó lipoinyección glútea y el segundo sirvió como control, sin lipoinyección.

Se consideraron como criterios de exclusión: pacientes menores de 18 años de edad, enfermedad de nervio periférico, cirugías previas a nivel lumbar, liposucción previa, cicatrices a nivel lumbar y/o glúteo.

Para el análisis de la sensibilidad, la región glútea se dividió en cuatro cuadrantes: superomedial, superolateral, inferomedial e inferolateral. Se evaluó en cada paciente la sensibilidad en los cuatro cuadrantes por el mismo examinador. Los parámetros analizados fueron roce, dolor, vibración, calor y frío. El roce se evaluó con una torunda de algodón; el dolor con una aguja de insulina; la vibración con un diapasón y las sensaciones de calor y frío con recipientes con agua caliente $\left(60^{\circ} \mathrm{C}\right)$ y hielos, respectivamente. Todos los resultados fueron descritos por los pacientes como positivos o negativos y se calculó la diferencia entre ambos grupos mediante $\chi^{2}$, así como el riesgo relativo.

La lipoinyección glútea se efectuó en los pacientes del primer grupo en el plano intramuscular con cánula de inyección roma de 4 $\mathrm{mm}$ de diámetro, a través de un acceso interglúteo. No se analizó el ángulo de inclinación de la cánula.

\section{RESULTADOS}

La presencia de alteraciones en la sensibilidad en el grupo con lipoinyección fue del 20\% (dos de 10 pacientes): una con insensibilidad al roce en el cuadrante superomedial izquierdo y otra en la región superolateral derecha. El promedio de volumen lipoinyectado en estas pacientes fue de $229 \mathrm{~mL} \pm 62$ en el lado derecho y 232 $\mathrm{mL} \pm 68$ en el lado izquierdo (Cuadro I).

En el grupo sin lipoinyección sólo una paciente presentó insensibilidad al roce en el cuadrante inferomedial derecho durante una semana (Cuadro II). Los demás parámetros evaluados fueron positivos en todos los pacientes.

\section{Cuadro I. Pacientes sometidas a liposucción más lipoinyección glútea.}

\begin{tabular}{|c|c|c|c|c|c|c|}
\hline Paciente & Sexo & Edad & $\begin{array}{l}\text { Volumen infiltrado } \\
\text { derecho }(\mathrm{mL})\end{array}$ & $\begin{array}{l}\text { Volumen infiltrado } \\
\text { izquierdo }(\mathrm{mL})\end{array}$ & Plano de infiltración & Complicación \\
\hline 1 & Femenino & 24 & 200 & 220 & Intramuscular & Ninguna \\
\hline 2 & Femenino & 36 & 320 & 350 & Intramuscular & $\begin{array}{l}\text { Insensibilidad al roce } \\
\text { y al dolor en cuadrante } \\
\text { superomedial izquierdo }\end{array}$ \\
\hline 3 & Femenino & 28 & 240 & 240 & Intramuscular & Ninguna \\
\hline 4 & Femenino & 33 & 200 & 200 & Intramuscular & $\begin{array}{l}\text { Insensibilidad al roce } \\
\text { y al dolor en cuadrante } \\
\text { superolateral derecha }\end{array}$ \\
\hline 5 & Femenino & 41 & 200 & 180 & Intramuscular & Ninguna \\
\hline 6 & Femenino & 26 & 350 & 350 & Intramuscular & Ninguna \\
\hline 7 & Femenino & 45 & 150 & 150 & Intramuscular & Ninguna \\
\hline 8 & Femenino & 37 & 200 & 200 & Intramuscular & Ninguna \\
\hline 9 & Femenino & 29 & 250 & 250 & Intramuscular & Ninguna \\
\hline 10 & Femenino & 30 & 180 & 180 & Intramuscular & Ninguna \\
\hline
\end{tabular}




\begin{tabular}{cccc} 
Cuadro II. Pacientes sometidas a liposucción sin lipoinyección glútea. \\
Paciente & Sexo & Edad & Complicación \\
\hline 1 & Femenino & 47 & Ninguna \\
2 & Femenino & 27 & Ninguna \\
3 & Femenino & 35 & Ninguna \\
4 & Femenino & 43 & Ninguna \\
5 & Femenino & 21 & Ninguna \\
6 & Femenino & 28 & Ninguna \\
7 & Femenino & 41 & Ninguna \\
8 & Femenino & 33 & Insensibilidad al roce en cuadrante \\
& & & ínferomedial derecho \\
9 & Femenino & 38 & Ninguna \\
10 & Femenino & 31 & Ninguna \\
\hline
\end{tabular}

No hubo diferencias significativas entre ambos grupos y el riesgo relativo fue de $2.0(\mathrm{Cl}$ 0.21-18.6), con una $p=0.54$.

\section{DISCUSIÓN}

Las lesiones nerviosas se pueden generar por diferentes mecanismos: tracción directa del nervio, daño mecánico, calor, ultrasonido o laceración directa. Otros mecanismos por los que se pueden presentar lesiones son debido al atrapamiento o compresión extrínseca o intrínseca de los nervios producido por material de sutura, tejido cicatricial, hematomas, compresión grasa y/o mala posición del paciente durante el transoperatorio. ${ }^{8,9}$

Las neuropatías relacionadas con los procedimientos de contorno corporal se deben en su mayoría a daño directo al nervio, compresión o tracción. Por eso, el cuidado de la posición del paciente, el adecuado acolchonamiento y conocimiento de la anatomía son cruciales para evitar las lesiones nerviosas.9,10

En la literatura se reporta la presencia de parestesias temporales en el sitio quirúrgico durante un periodo aproximado de una semana, incluso se menciona el uso de fajas de compresión postoperatorias como causal de hipoestesia durante un periodo de 48 horas aproximadamente..$^{8,9}$

En la lipoinyección glútea se ha descrito específicamente lesiones nerviosas que incluyen lesión al nervio ciático por lesión directa o compresión extrínseca por el tejido lipoinyectado. La mayor parte de las lesiones reportadas se presentan como parestesias o hipoestesias leves, con una duración aproximada de siete a 15 días; sin embargo, existen reportes de lesiones severas, como la infiltración directa del nervio ciático. ${ }^{9-13}$

En este estudio, el $20 \%$ de las pacientes sometidas a lipoinyección glútea tuvieron alteraciones de la sensibilidad a seis meses de la intervención quirúrgica y fue mayor que los reportes previos; no obstante, se requieren estudios prospectivos para tener una evaluación más exacta. ${ }^{10-13}$

\section{CONCLUSIÓN}

En la región glútea hay nervios motores y sensitivos en riesgo durante los procedimientos de contorno corporal, especialmente en la lipoinyección glútea.

La insensibilidad glútea después de un procedimiento estético de lipoinyección se debe vislumbrar y plantear como una complicación relativamente frecuente. Dicha insensibilidad secundaria a un procedimiento estético puede traer consecuencias catastróficas, tanto para el paciente como para el cirujano, motivo por el cual el conocimiento detallado de la anatomía y el cuidado del paciente en el transoperatorio son cruciales para evitar lesiones nerviosas.

\section{REFERENCIAS}

1. Rosique RG, Rosique MJ, De Moraes CG. Gluteoplasty with autologous fat tissue: experience with 106 consecutive cases. Plast Reconstr Surg 2015; 135 (5): 1381-1389.

2. Avendaño-Valenzuela G. Contorno de la región glútea. Conceptos actuales y propuesta de clasificación. Cir Plast Iberolatinoam 2010; 36 (1): 239-245.

3. Gonzalez-Ulloa M. Gluteoplasty: a ten year report. Aesthet Plast Surg 1991; 15: 85-91.

4. De la Peña JA. Subfascial technique for gluteal augmentation. Aesthet Surg J 2004; 24: 265-273.

5. González R. Remodelación de nalgas: iprótesis o lipoinjerto? Cir Est Plast 2009; 10: 10.

6. Cuenca-Guerra R, Lugo-Beltran I. Beautiful buttocks: characteristics and surgical techniques. Clin Plast Surg 2006; 33 (3): 321.

7. Porto Da Rocha R. Surgical anatomy of the gluteal region's subcutaneous screen and its use in plastic surgery. Aesthet Plast Surg 2001; 25 (2): 140-144.

8. Condé-Green A, Kotamarti V, Nini KT et al. Fat grafting for gluteal augmentation: a systematic review of the 
literature and meta-analysis. Plast Reconstr Surg 2016; 138 (3): 437e-446e.

9. Sinno S, Chang JB, Brownstone ND, Saadeh PB, Wall $S \mathrm{Jr}$. Determining the safety and efficacy of gluteal augmentation: a systematic review of outcomes and complications. Plast Reconstr Surg 2016; 137 (4): 1151-1156.

10. de la Concha BE, Telich TJ, Cárdenas-Mejía A. Lesiones nerviosas en procedimientos de cirugía estética: incidencia, prevención y manejo. Cir Plast 2015; 25 (3): 168-176.

11. Cardenas-Mejia A, Martínez JR, León D, Taylor JA, Gutierrez-Gomez C. Bilateral sciatic nerve axonotmesis after gluteal lipoaugmentation. Ann Plast Surg 2009; 63 (4): 366-368.

12. Oranges CM, Tremp M, Di Summa PG, Haug $M$, Kalbermatten DF, Harder $Y$ et al. Gluteal augmentation techniques: a comprehensive literature review. Aesthetic Surgery Journal 2017; 37 (5): 560-569.

13. Bruner TW, Roberts TL 3rd, Nguyen K. Complications of buttocks augmentation: diagnosis, management, and prevention. Clin Plast Surg 2006; 33 (3): 449-466.

Correspondencia:

Dr. André Víctor Baldin

$2^{\circ}$ piso - Cirugía Plástica y Reconstructiva, Hospital General «Dr. Manuel Gea González».

Av. Calzada de Tlalpan Núm. 4800,

Col. Sección XVI, 14080,

Tlalpan, Ciudad de México.

Teléfono: 4000 3000, ext. 3721

E-mail: andre.baldin03@gmail.com 